
\title{
R Reserach S Suare \\ The Role of Genetic Variation in Maize Response To Beneficial Endophytes
}

Corey Robert Schultz ( $\nabla$ crs68219@uga.edu )

University of Georgia https://orcid.org/0000-0003-4739-3238

Kamaya Brantley

University of Georgia

Jason G Wallace

University of Georgia

\section{Research Article}

Keywords: growth promoting, crop-microbe interaction, quantifying variance

Posted Date: January 10th, 2022

DOI: https://doi.org/10.21203/rs.3.rs-1189677/v1

License: (c) (i) This work is licensed under a Creative Commons Attribution 4.0 International License.

Read Full License 


\section{Abstract}

Growth-promoting endophytes have great potential to boost crop production and sustainability. There is, however, a lack of research on how differences in the plant host affect an endophyte's ability to promote growth. We set out to quantify how different maize genotypes respond to specific growth-promoting endophytes. We inoculated genetically diverse maize lines with three different known beneficial endophytes: Herbaspirillum seropedicae (a gram-negative bacteria), Burkholderia WP9 (a gram-negative bacteria), and Serendipita vermifera Subsp. bescii (a Basidiomycota fungus). Maize seedlings were grown for 3 weeks under controlled conditions in the greenhouse and assessed for various growth promotion phenotypes. We found Herbaspirillum seropedicae to increase chlorophyll content, plant height, root length, and root volume significantly in different maize genotypes, while Burkholderia WP9 did not significantly promote growth in any lines under these conditions. Serendipita bescii significantly increased root and shoot mass for 4 maize genotypes, and growth promotion correlated with measured fungal abundance. Although plant genetic variation by itself had a strong effect on phenotype, its interaction with the different endophytes was weak, and the endophytes rarely produced consistent effects across different genotypes. This genome-by-genome interaction indicates that the relationship between a plant host and beneficial endophytes is complex, and it may partly explain why many microbebased growth stimulants fail to translate from laboratory settings to the field. Detangling these interactions will provide a ripe area for future studies to understand how to best harness beneficial endophytes for agriculture.

\section{Introduction}

Food security is critical to modern global society. However, problems such as soil degradation, climate change, and a growing population will challenge our global food supply in the 21st century [Affairs 2015, Ray et al. 2013]. Improving crop yield by even small percentages results in massive increases in product and reduces the environmental burden of production. Biostimulants are a class of agricultural inputs based on either living organisms or products derived from them, and the use of biostimulants is touted as a sustainable way to improve yield, reduce soil degradation, and provide other ecological benefits [Majeed et al. 2018].

A common class of biostimulants involves the use of endophytes, microbes that live inside plants' tissue [Wallace and May 2018]. Previous studies have identified many ways that endophytes impact their hosts, such as by supplying nutrients (including nitrogen) [Baldotto et al. 2012, Boddey et al. 1991, Matsumura et al. 2015, Young et al. 2013], increasing stress resistance [Akhtar et al. 2015, Arachevaleta et al. 1989, Cohen et al. 2009, Naveed et al. 2014] and increasing crop growth and yield [Akhtar et al. 2015, Canellas et al. 2012, Hungria et al. 2010, Young et al. 2013]. Endophytes can stimulate plant growth in a variety of ways, such as by out-competing pathogens [Sobowale et al. 2007], producing antimicrobial compounds [Mousa et al. 2015, Shehata et al. 2016,Shehata et al. 2017], synthesizing/increasing phytohormones and secondary metabolites [Cohen et al. 2009, Kumara et al. 2013, Rivas-Franco et al. 2020], and mitigating stress [[Akhtar et al. 2015, Arachevaleta et al. 1989, Cohen et al. 2009, Naveed et al. 2014]. 
The biostimulant market is projected to be worth 11 billion USD by 2027 [Biostimulants Market 2021]. Many such treatments are already commercially available in the form of foliar sprays, soil treatments, and seed treatments. Both startup companies and large corporations like Bayer and Syngenta are heavily investing in biostimulants [Biostimulants Market 2021], and trends in the scientific literature indicate growing interest in endophyte growth promotion in the public sector as well [Rho et al. 2017]. Despite this, it is incredibly challenging to bring a biostimulant from the lab to market [Parnell et al. 2016, Timmusk et al. 2017]. Many microbes that appear promising in the lab do not produce reliable effects in the field. Although many factors are probably responsible for this issue, one that has received less attention is how different plant genotypes respond to beneficial endophytes.

Several groups have shown that the effects of beneficial microbes vary across genotypes [Alves et al. 2014, Arujo et al. 2013, Brusamarello-Santos et al. 2017], but there has been relatively little quantification of this effect and almost no exploration of the underlying mechanisms. For example, an increase in maize yield due to bacteria in the genus Herbaspirillum depended on both the endophyte strain and the maize variety, with Herbaspirillum seropedicae (ZAE 94) increasing biomass in less than a third of commercial maize genotypes tested [Alves et al. 2014]. One of the few studies investigating this interaction traced the variation in Arabidopsis' response to growth-promoting rhizobacteria to several candidate genes, including genes involved in plant-growth processes like transporters and metabolism [Wintermans et al. 2016]. Understanding the nature and extent of this variation could provide a way to improve the development and use of biostimulants in agriculture.

Maize is one of the most important crops in global agriculture. Over a billion tonnes of maize were produced worldwide in 2019 [FAOSTAT 2021]. Although not investigated in detail, previous research has shown that the same endophyte impacts different maize inbreds to different degrees, and these differences are likely due to differences in the host's genetics [Alves et al. 2014, Arujo et al. 2013, Brusamarello-Santos et al. 2017, Montanez et al. 2012, Naveed et al. 2014, Riggs et al. 2001, Walters et al. 2018]. Despite the number of studies that mention such genotype-by-microbe interactions, few if any of them quantify the size of the effect, so it is difficult to determine just how important of a factor this variation is. Understanding and effectively utilizing these interactions would be a step towards increasing yield in a sustainable fashion.

In this study we aimed to quantify the effect maize genotype has on host-endophyte interaction. In a series of three experiments, we inoculated diverse maize lines with one of three different endophytes (Herbaspirillum seropedicae, Burkholderia WP9, and Serendipita bescii) and quantified the resulting changes in phenotype.

Herbaspirillum seropedicae is a well-studied gram-negative, growth-promoting endophyte that is commonly used to study nitrogen fixation in symbioses with grasses [Alves et al. 2014, Canellas et al. 2012, Dall'Asta et al. 2018]. It has a broad host range and can colonize sugarcane, rice, wheat, and maize, where it can act as a biofertilizer [Boddey et al. 1991, Majeed et al. 2018, Rosenblueth et al. 2018]. In addition to fixing nitrogen, $H$. seropedicae can solubilize minerals and produce phytohormones [Monteiro 
et al. 2012]. It has been shown that maize yield, metabolite content, and shoot dry weight depend upon the genotype of both the Herbaspirillum inoculant, as well as the genotype of the maize host [Alves et al. 2014, Arujo et al. 2013, Brusamarello-Santos et al. 2017].

Burkholderia is bacterial genus containing well-studied growth promoting endophytes. Different species have been shown to enhance growth, yield, and disease resistance [Young et al. 2013] and aid in the uptake of phosphate and nitrogen [Estrada et al. 2012, Young et al. 2013]. Burkholderia WP9 was isolated from black cottonwood and has nitrogen fixing abilities [Doty et al. 2009].

Serendipita bescii is a Basidiomycota fungus, and fungi in this genus are known to associate with many different plant species as an endomycorrhizae [Ray et al. 2018]. Originally recognized as orchid mutualists, Serendipita fungi also promote growth in a number of different plants, including switchgrass [Ray et al. 2015]. It is hypothesized that when serendipitoid fungi colonize root systems, they break down organic manner in the soil and make these nutrients available to the plant [Craven and Ray 2019].

For each microbe, we determined its effect on seedling phenotypes of diverse maize varieties and quantified the effect of maize genetics, microbes, and their interaction. These results provide a firm estimate of the degree to which different maize genotypes respond to beneficial endophytes, and how genetic variation among these lines modulates that response.

\section{Materials And Methods Experimental Design}

Maize genotypes were selected from among the Goodman-Buckler diversity panel [Flint-Garcia et al. 2005], with most also being founders of the maize Nested Association Mapping population [Mcmullen et al. 2009] (Table 3). For space reasons, each experiment was subdivided into a series of "grows" including only a subset of plant genotypes. Within each grow, plants were arranged in a randomized complete block design with 5 reps; in a few cases seedlings died after germination leaving that genotype with four reps. 
Table 1

Maize germplasm

\begin{tabular}{|lll|}
\hline Maize Genotype & GRIN Accession & Experiment(s) Used \\
\hline A635 & PI 693329 & $1,2,3$ \\
\hline B73 & PI 5504073 & 1,3 \\
\hline B97 & PI 564682 & 1,3 \\
\hline CML 52 & PI 595561 & 1,3 \\
\hline CML 103 & Ames 27081 & 1,3 \\
\hline CML 228 & Ames 27081 & 1,2 \\
\hline CML 333 & Ames 27101 & 3 \\
\hline HP301 & PI 587131 & 3 \\
\hline KI3 & Ames 27123 & 1,3 \\
\hline KI11 & Ames 27124 & 2,3 \\
\hline M017 & PI 558532 & 1 \\
\hline MS71 & PI 587137 & $1,2,3$ \\
\hline NC350 & Ames 27171 & $1,2,3$ \\
\hline P39 & Ames 28186 & 2,3 \\
\hline TX303 & Ames 19327 & 1,3 \\
\hline
\end{tabular}

\section{Seed Sterilization and Plant Growth}

Seeds were surface sterilized for five minutes using $50 \mathrm{~mL}$ sterile $\mathrm{H}_{2} \mathrm{O}, 50 \mathrm{~mL}$ of bleach(Clorox), and three drops of Tween $20(V W R)$. Seeds were rinsed five times with $100 \mathrm{~mL}$ of sterile water, then immersed in a $60^{\circ} \mathrm{C}$ water bath for 15 minutes to kill existing endophtytes [Bacon C.W. 1994]. Seeds (in water) were then allowed to cool and imbibe for 1 hour before placing 10 seeds equidistant from each other in an autoclaved magenta box with $15 \mathrm{ml}$ nutrient agar (1x Hoagland solution [bioWorld 30630038-5] + 15g/L of agar [Caisson Labs])). The box was then parafilmed shut, and the seeds were allowed to germinate for seven days. After 7 days, seedlings were moved to the greenhouse and planted $4 \mathrm{~cm}$ deep in 2.37 liter pots filled with autoclaved Professional Growing Mix Fafard 3B/Metro-Mix 830 (Sungro Horticulture) and inoculated as described below. Pots were watered three times a week, and plants were grown for an additional 21 days before they were harvested.

\section{Bacterial growth and inoculation}

Experiment 1: H. seropediceae (ATTC 35892) was grown from a single colony in nutrient broth at $24^{\circ} \mathrm{C}$ for 48 hours to an OD of $\sim 0.8$. Germinated seeds were placed into the autoclaved soil and inoculated with 
$2 \mathrm{~mL}$ of culture or sterile nutrient broth (control) before being covered by soil. No additional water was applied for 2 days to allow for colonization.

Experiment 2: Burkholderia WP9 (Sharon Doty, University of Washington) was grown from a single colony in nutrient broth at $24^{\circ} \mathrm{C}$ for 48 hours to an OD of $\sim 0.8$. Autoclaved soil was inoculated with $200 \mathrm{~mL}$ of culture/kg of soil, or with sterile nutrient broth for controls, before being placed into individual pots and germinated seedlings planted as above.

Experiment 3: Serendipita bescii (Kelly Craven, Noble Research Institute) was pre-inoculated onto clay bentonite particles [Ray et al. 2015, Rat et al. 2018] by collaborators at the Noble Research Institute. Based on their recommendation, soil was placed into pots a week before sowing and thoroughly washed with water 5 times to leech minerals and nutrients from the media. $100 \mathrm{~g}$ of clay particles (inoculated or control) were placed in a depression in the soil, with the germinated seed then placed on top and covered.

After 21 days, the above- and below-ground portions of each plant were separated with a sterile razor blade and frozen at $-80^{\circ} \mathrm{C}$ for further phenotyping.

\section{PCR confirmation of colonization}

For each experiment, endophyte colonization was confirmed by PCR. About $0.5 \mathrm{~g}$ of washed root was collected using a sterile razor blade $\sim 8 \mathrm{~cm}$ from the base of the root. Samples were placed into a $2 \mathrm{~mL}$ microtube with a sterile metal ball (Daisy BBs) and placed into a GenoGrinder 2010 at 1400 RPMs for 5 minutes. DNA was extracted with a Quick-DNA Fungal/Bacterial kit (Zymo) and DNA quality and concentration checked via Infinite M200 Pro (TECAN). PCR was run on each sample using the specific endophyte primers (Additional File 1 ) and run on a 1\% agarose gel to confirm colonization. Noninoculated plants served as controls for greenhouse contamination. PCR program was as follows: $30 \mathrm{~s}$ at $95^{\circ} \mathrm{C}$, followed by 30 cycles of $15 \mathrm{~s}$ at $95^{\circ} \mathrm{C}, 60 \mathrm{~s}$ at $59^{\circ} \mathrm{C}, 30 \mathrm{~s}$ at $68^{\circ} \mathrm{C}$, ending with $5 \mathrm{~m}$ at $68^{\circ} \mathrm{C}$ and hold at $4^{\circ} \mathrm{C}$.

\section{Phenotyping Methods}

Plant Height was measured from the soil line to the tip of the longest/tallest leaf when held upright, and was recorded every week. This method is thus is a combination of plant height and leaf length, and was used to control for different leaf angles.

Chlorophyll Quantum Yield was measured using a Flouropen FP 100 (Photon Systems Instruments). Measurements were taken from halfway up the most mature leaf on the plant. Three measurements were taken at the same location and averaged.

Leaf Area: The newest mature leaf was gently removed at the collar. Leaves were laid flat and pinned to a white surface next to a 1 "-square size marker and a paper with sample identifying information (name \& date). Images of each leaf were quantified with EasyLeafArea [Easlon et al 2014], with the following batch parameters: Leaf minimum Green RGB value 15, Leaf Green Ratio (G/R) 1.06, Leaf Green Ratio (G/B) 1.08, Scale Minimum Red RGB value 50, Scale Red Ratio 1.96, Scale area $\left(\mathrm{cm}^{2}\right)=6.5 \mathrm{~cm}^{2}$. 
Shoot Mass: The entire aboveground portion of the plant, including the leaf removed to measure leaf area, was dried in a forced-air oven at $37.7^{\circ} \mathrm{C}$ for 48 hours before being weighed on a precision balance (VWR 164AC).

Roots: Frozen roots were removed from the freezer and washed with warm sterile water while gently rubbing to remove as much soil as possible without damaging the root system. Root length was measured from the base to the end of the longest root. Root volume was measured by placing the washed \& dried roots into a 20-ml graduated cylinder half-filled with water and recording the displacement volume as the roots were submerged. Dry root mass was measured on a precision balance (VWR 164AC) after air-drying samples for 48 hours at $37.7^{\circ} \mathrm{C}$ in a forced-air drier.

qPCR for fungal biomass: For Experiment 3 only, relative fungal biomass was estimated using the $2^{\triangle \triangle C t}$ method [Livak and Schmittgen 2001] to compare the amount of fungal ITS3 to maize CDK DNA in each sample. Extracted maize root DNA (the same used to confirm colonization, above) was diluted to 12 ng/uL using an Infinite M200 Pro (TECAN). qPCR was performed using primers specific for the Serendipita ITS3 gene [Ray et al. 2015] or the maize CDK (cycline-dependent kinase housekeeping gene) [Lin et al. 2014] (Table S1). Reactions were performed using SYBR Green I Master Mix (Roche) and the manufacturer's recommended protocol (pre-incubation at $95^{\circ} \mathrm{C}$ for $5 \mathrm{~m}, 45$ cycles of amplification for $10 \mathrm{~s}$ at $95^{\circ} \mathrm{C}, 49.6^{\circ} \mathrm{C}(\mathrm{ITS})$ or $59.3^{\circ} \mathrm{C}(\mathrm{CDK})$ for $18 \mathrm{~s}$, and $30 \mathrm{~s}$ at $\left.72^{\circ} \mathrm{C}\right)$. A single melting curve was performed, with 8 acquisitions $/{ }^{\circ} \mathrm{C}$. Reactions were run on a Roche LightCycler 480 , with two technical replicates for each sample. $2^{\Delta \Delta C t}$ values were generated from threshold crossing $(\mathrm{Ct})$ values, and then log transformed to visualize $S$. bescii colonization for each maize genotype in $\mathrm{R}$.

\section{Statistics}

All statistics were run in R [R Core Team]. Due to space constraints, groups of genotypes ("grows") had to be planted separately throughout each experiment; since these grows were completely confounded with the plant genotype, they were not included in subsequent analyses. ANOVA was performed by fitting a linear model of Phenotype Genotype + Condition + Genotype:Condition, where "Condition" represents either inoculated or control. The resulting ANOVA table was used to calculate the fraction of total variation contributed by each component and its statistical significance. The Genome x Genome $(G \times G)$ interaction represents the fraction of variation explained after accounting for the main effects of maize genotype and the general effect of inoculation. $G x G$ is calculated as $V_{G C} /\left(V_{G C}+V_{e}\right)$, where $V_{G C}$ is the variation due to genotype-by-condition interaction, and $V_{e}$ is the residual (error) variance. Significant growth differences were determined using a Welch's two-sided t-test between inoculated and control plants of the same genotype. Levene's test was used to check for homogeny of variance for each phenotype.

\section{Results}

We tested three separate endophytes for growth promoting abilities in maize inbred lines in three separate greenhouse experiments. Due to space constraints, experiments had to be subdivided into Grows, which 
contained all of the replicates for a genotype. The experimental design was kept identical for grows within the same experiment, with planting date being the only difference. All tested phenotypes are listed in Table 1. In these experiments, "genome-by-genome interaction" (GxG) refers to how much variation was due to the specific combination of maize variety and endophyte inoculation. Functionally, GxG represents the differences in how maize varieties respond to an endophyte, so that the larger $G \times G$ is the stronger the differences are among maize varieties.

\section{Experiment 1}

Experiment 1 used the gram-negative bacterium Herbaspirillum seropediceae Z67, with liquid culture applied directly to germinated seeds. Phenotypic variation was largely due to the plant genotype (Figure 1), as was expected given the high genetic variation in maize. Inoculation with $H$. seropediceae was not a significant source of variation for any phenotype, and GxG interaction was only statistically significant for root volume ( $p=.05$; Figure 1$)$. Root volume had the highest $G \times G$ for the entire study $(0.243$, after accounting for the main effects of genotype and endophyte; Table 1). When looking at individual genotypes instead of the experiment as a whole, $H$. seropediceae increased chlorophyll content, plant height, and root volume (Table 3), though only for 1 maize genotype in each case. Herbaspirillum increased growth, though generally only for a couple of maize genotypes. There was also an interesting trend where $H$. seropediceae increased root length in A635 but decreased it in CML52, though it did not reach statistical significance. 
Table 2

Genome by Genome Interaction Calculations

\begin{tabular}{|lll|}
\hline Endophyte & Trait & GxG Interaction (0-1) \\
\hline Experiment 1 & & \\
\hline Herbaspirillum & Chlorophyll & .057 \\
\hline Herbaspirillum & Plant Height & 0.091 \\
\hline Herbaspirillum & Leaf Area & 0.155 \\
\hline Herbaspirillum & Root Length & 0.157 \\
\hline Herbaspirillum & Root Volume & 0.243 \\
\hline Experiment 2 & & \\
\hline Burkholderia & Plant Height & 0.218 \\
\hline Burkholderia & Leaf Area & 0.232 \\
\hline Burkholderia & Root Length & 0.071 \\
\hline Burkholderia & Root Volume & 0.027 \\
\hline Experiment 3 & & \\
\hline Serendipita & Plant Height & 0.178 \\
\hline Serendipita & Root Length & 0.157 \\
\hline Serendipita & Root Mass & 0.144 \\
\hline Serendipita & Shoot Mass & 0.222 \\
\hline
\end{tabular}

Table 2. Genome x Genome interaction calculations. GxG were calculated as the fraction of phenotypic variance due to Genotype:Inoculation interaction after accounting for the main effects of maize genotype and inoculation.

\section{Experiment 2}

Experiment 2 used the gram-negative bacteria Burkholderia sp. WP9, with liquid culture applied directly to bulk soil immediately before adding seeds. Again, plant genotype was statistically significant for most measured phenotypes while endophyte inoculation by itself was not significant for any (Figure 1). Plantendophyte interaction was statistically significant for both Plant Height and Leaf Area, with GxG interaction scores of $\sim 0.2$ (Table 1). Although Burkholderia WP9 reportedly boosts maize growth (Sharon Doty, personal communication), we saw no statistically significant growth promotion for any individual phenotypes in any lines that were tested (Table 2). The fact that GxG interaction overall is significant while no individual genotypes are is probably due to the greater statistical power when looking at the experiment as a whole. 
Table 3

Significant Growth Differences

\begin{tabular}{|lll|}
\hline Trait & \multicolumn{1}{c}{$\mathbf{P}<0.1$} & $\mathrm{P}<0.05$ \\
\hline Experiment 1 & - H. Seropedicaea & \\
\hline Chlorophyll & CML103(+) & B73(+) \\
\hline Plant Height & - & Mo17(+) \\
\hline Leaf Area & - & - \\
\hline Root Length & CML52(-), A635(+) & - \\
\hline Root Volume & TX303(+) & CML228(+) \\
\hline Experiment 2 - Burkholderia WP9 & \\
\hline Plant Height & - & - \\
\hline Leaf Area & CML228(-) & - \\
\hline Root Length & - & - \\
\hline Root Volume & - & - \\
\hline Experiment 3 - S. bescii & \\
\hline Plant Height & - & TX303(+), CML52(+) \\
\hline Root Length & B73(-), CML103(-), A635(+) & - \\
\hline Root Mass & KI11(+) & P39(+), CML52(+) \\
\hline Shoot Mass & - & P39(+), NC350(+), TX303(+) \\
\hline
\end{tabular}

Table 3. Genotypes that have significant growth promotion for a measured phenotype. Significance was determined by a two-sided t-test of control versus inoculated plants (see Methods). The direction of growth (increase or decrease) is indicated by a "+" or "-" sign after each genotype.

\section{Experiment 3}

Experiment 3 used the Basidiomycota fungus Serendipita bescii, which was pre-inoculated on sterile clay particles that were placed directly under the germinated seed in the soil. S. bescii showed the strongest growth-promoting effects of the three endophytes, with a significant main effect of inoculation for all four measured phenotypes (Figure 1). S. bescii increased growth in a number of genotypes (Table 2; Figure 2), though GxG interaction was not significant in the experiment overall (Figure 1), possibly due to the high variance within some of the lines. A particularly interesting contrast in this experiment involves the maize lines CML52, NC350, P39, and TX303 (Table 2). Inoculation with $S$. bescii increased only the aboveground biomass of NC350 and TX303, only the belowground biomass of CML52, and both traits for P39 (Figure 2). 
These differences in phenotype response could be due to differences in endophyte colonization, as has been shown for other grasses [Faville et al. 2015, Khan et al. 2016]. We quantified the colonization of Serendipita using qPCR of the Serendipita fungal ITS3 [Ray et al. 2015] normalized against the maize housekeeping gene CDK [Lin et al. 2014] (Figure 3). Higher levels of Serendipita colonization coincided with increased growth in the greenhouse. Maize lines P39, NC350, TX303, and CML52 had high Serendipita in their respective grows, and showed growth promotion for at least one phenotype with a pvalue < 0.05 (Table 2); two other maize genotypes (A635 and KI11) also had high S. bescii abundances but less significant growth promotion ( $p<0.1$; Table 2$)$. Of the remaining lines, MS71 and CML333 showed no growth promotion, while CML103 and B73 had a nonsignificant trend $(p \sim 0.07)$ toward growth inhibition.

\section{Discussion}

Our results in this study highlight how three potentially growth-promoting endophytes affect genetically diverse maize. When inoculated with Herbaspirillum seropedicae (Experiment 1), we found several maize genotypes showed increased growth in one of several phenotypes (Chlorophyll content, plant height, root length, and root volume, Table 2). Interestingly, each genotype showed an increase in a specific phenotype, and genome-by-genome interaction played a significant role only for root volume (Figure 1). Experiment 2, examining Burkholderia WP9, indicated significant GxG interaction for two phenotypes (plant height and leaf area), though an examination of individual varieties did not show statistically significant effects, probably due to the smaller sample size. Since other studies have shown that this isolate promotes growth in both rice [Khan et al. 2016] and maize (Sharon Doty, personal communication), it may be worth additional investigation. Experiment 2 did show that some maize genotypes were slightly hindered by the endophyte, while some received slight growth promotion, highlighting the importance of genotype interactions. Of the genotypes inoculated with Serendipita bescii (Experiment 3), one (P39) showed growth increases both above and below ground (Figures 2 \& 3), while three other genotypes (NC350, CML52 and TX303) experienced increases in only one of the two categories. Similarly to Burkholderia, inoculation with Serendipita decreased the root length of two maize genotypes (B73 and CML103), further emphasizing the potential for unique reactions to endophyte treatment among different genotypes. Serendipita was the only endophyte to show a main effect of inoculation across all phenotypes (Figure 1), meaning it was the only one of the three endophytes to have a relatively consistent effect on hosts regardless of genotype.

Not only did Serendipita have the most consistent effect on hosts, we also showed that the size of this effect may be related to the plants colonization level (Figure 4). This indicates that stronger growthpromotion phenotypes may be a direct consequence of higher endophyte loads. We attempted to find other examples of this trend in the literature, but there are surprisingly few studies that directly compare endophyte colonization with growth promotion. One exception involves Epichloë fungal endophytes, whose biomass has been shown to correlate with the amount of protective alkaloid compounds in their grass hosts [Cagnano et al. 2020, Faville et al. 2015]. Further testing is needed to confirm if endophyte 
biomass amounts have a direct influence on growth promotion, and to uncover the mechanisms and genetic diversity behind these interactions in maize.

This data does not provide insight into the molecular mechanisms of genome-by-genome interaction, but interest in this field is quickly growing. Prior work indicates that these interactions could be impacted by a number of metabolites and pathways. For example, diverse maize genotypes respond differently to microbe-associated molecular patterns (MAMPs), and as a consequence show significant differences in reactive oxygen species, nitric oxide production, and defense gene expression [Wang et al. 2021, Zhang et al. 2017]. It has been suggested that Herbaspirillum may regulate the ROS pathway differently in diverse maize roots [Brusamarello-Santos et al. 2017]. Many endophytes produce phytohormones [Cohen et al. 2009, Wallace and May 2018] and volatile organic compounds [Kumara et al. 2013, Monteiro et al. 2012], which directly impact growth promotion and stress resistance. Future studies may be able to shed light on how host genetic diversity impacts these molecular interactions.

A chief goal of this research was to quantify "Genome-by-Genome interaction" in this system, meaning changes in phenotype that depend on both the maize genotype and the specific endophyte. The GxG interactions we identified explain relatively little phenotypic variance, even after factoring out the effect of maize genotype (Table 1). The strongest GxG effects explain $\sim 20-25 \%$ of residual variance after accounting for genotype, although most are half this or less. Looked at another way, GxG interaction generally explained only $1 / 10$ th of the variance that maize genotype did, meaning that the effect was 10 times weaker than the effect of plant genetics. This small effect size may make it challenging to attempt to dissect the underlying genetic components that affect plant-microbe interactions, a conclusion shared by a recent review of GWA studies [Bergelson et al. 2021]. One should keep in mind, however, that the maize varieties in this study were specifically chosen for their high diversity. Diversity in elite breeding programs is generally much lower, so endophyte inoculation may have relatively larger effects within elite material.

These data demonstrates that maize genotype does have a significant impact on endophyte-mediated growth promotion. In addition, all three endophytes showed indications of decreased growth in at least one maize line, but none of these lines were the same. On the other hand, of the nine maize genotypes tested with both $H$. seropedicae and S. bescii, only one (TX303) showed statistically significant growth promotion for both endophytes. Interestingly, this promotion was belowground (Root Volume) for $H$. seropedicae and above ground (Plant Height) for $S$. bescii. This may indicate that these endophytes are activating different pathways or altering the expression of different genes.

\section{Conclusion}

Our interactions with maize producers in the US indicates that they are both interested in biologicals and concerned about their efficacy. Our findings show how different maize inbreds react to the same beneficial microbe. These findings provide insight into the range of responses between plants and microbes in an agricultural setting, and are especially important for groups developing new bioinoculates 
and biofertilizers. The high variability across genotypes may partly explain why many beneficial microbes reported in the literature fail to translate to field production. (This is apart from logistical factors such as scalability, shelf life, ease of use, and compatibility with existing formulation [Parnell et al. 2016, Timmusk et al. 2017], all of which also play a role.) These results imply that new microbial-based products for agriculture should be screened against diverse genotypes early in the process so as to weed out microbes with variable effects. Conversely, it may be possible to eventually tailor microbes to interact well with specific genotypes and deliver them as a specific plant-microbe combination (using seed treatments, for example). Further studies will show how these interactions occur and how we can best harness them to improve global agriculture.

\section{Declarations}

\section{Acknowledgments}

We would like to thank Dr. Sharon Doty (University of Washington) for supplying the Burkholderia WP9 for this experiment, and Drs. Kelly Craven and Prasun Ray (Noble Research Institute) for supplying the Serendipita bescii inoculum.

\section{Funding}

Funding for this experiment was provided by the University of Georgia and the Foundation for Food and Agriculture Research (FFAR).

\section{Ethics approval and consent to participate}

Not applicable

\section{Consent for publication}

Not applicable

\section{Availability of data and materials}

The datasets generated and analyzed during the current study, along with all code are available at https://github.com/wallacelab/paper-schultz-endophytes-2021

\section{Conflict of Interest}

The authors have no relevant financial or non-financial interests to disclose.

The authors have no competing interests to declare that are relevant to the content of this article.

All authors certify that they have no affiliations with or involvement in any organization or entity with any financial interest or non-financial interest in the subject matter or materials discussed in this manuscript. 
The authors have no financial or proprietary interests in any material discussed in this article.

\section{Authors' contributions}

J.W. conceived and supervised the work. C.S. performed the experiments and data analysis. K.B. assisted with Experiment 2. J.W. and C.S. co-wrote the manuscript. All authors reviewed the results and approved the final version of the manuscript.

\section{References}

1. Affairs UN (2015) D.o.E.a.S., World Population Prospects: The 2015 Revision, Key Findings and Advance Tables. Working Paper No ESA/WP.241,

2. Akhtar S, Saleem (2015) Interactive Effect of Biochar and Plant Growth-Promoting Bacterial Endophytes on Ameliorating Salinity Stress in Maize.. " Funct Plant Biol 42(8):770. doi:10.1071/fp15054

3. Alves G, Cavalcanti (2014), pp. 307-321., Differential Plant Growth Promotion and Nitrogen Fixation in Two Genotypes of Maize by Several Herbaspirillum Inoculants.. " Plant Soil 387:1-2. doi:10.1007/s11104-014-2295-2

4. Arachevaleta M et al (1989) Effect of the Tall Fescue Endophyte on Plant-Response to Environmental-Stress. Agronomy Journal 81(1):83-90

5. Araujo FF, De et al (2013) "Híbridos e Variedades De Milho Submetidos à Inoculação De Sementes Com Herbaspirillum Seropedicae." Semina: Ciências Agrárias, vol. 34, no. 3, doi:10.5433/16790359.2013v34n3p1043

6. BACON CW (1994) A corn seedling assay for resistance tofusarium moniliforme. Plant Dis 78(3):302. doi:10.1094/pd-78-0302

7. Baldotto MA, Borges Baldotto LE, Santana RB, Marciano CR (2012) Initial performance of maize in response to NPK fertilization combined with Herbaspirillum seropedicae.Revista Ceres59

8. Bergelson J, Brachi B, Roux F, Vailleau F (2021) Assessing the potential to harness the microbiome through plant genetics. Curr Opin Biotechnol 70:167-173. https://doi.org/10.1016/j.copbio.2021.05.007

9. Biostimulants Market. Market Research Firm. (n.d.). https://www.marketsandmarkets.com/MarketReports/biostimulant-market-1081.html

10. Boddey RM et al (1991) Biological Nitrogen-Fixation Associated with Sugar-Cane. Plant and Soil 137(1):111-117

11. Brusamarello-Santos L, Cristina (2017) Metabolic Profiling of Two Maize (Zea Mays L.) Inbred Lines Inoculated with the Nitrogen Fixing Plant-Interacting Bacteria Herbaspirillum Seropedicae and Azospirillum Brasilense.. " PLoS ONE 12(3). doi:10.1371/journal.pone.0174576

12. Cagnano G, Lenk I, Roulund N, Jensen CS, Cox MP, Asp T (2020) Mycelial biomass and concentration of loline alkaloids driven by complex population structure in Epichloë uncinata and meadow fescue 
(Schedonorus pratensis). Mycologia 112(3):474-490.

https://doi.org/10.1080/00275514.2020.1746607

13. Canellas L, Pasqualoto (2012), pp. 119-132., A Combination of Humic Substances and Herbaspirillum Seropedicae Inoculation Enhances the Growth of Maize (Zea Mays L.).. " Plant Soil 366:1-2. doi:10.1007/s11104-012-1382-5

14. Cohen AC, Travaglia CN, Bottini R, Piccoli PN (2009) Participation of abscisic acid and gibberellins produced by endophytic Azospirillum in the alleviation of drought effects in maize. Botany 87:455462

15. Craven KD, Ray P (2019) More than Serendipity: The Potential to Manage Soil Carbon and Emissions While Promoting Low-Input Agriculture with Serendipitoid Mycorrhizae.. " Phytobiomes Journal 3(3):161-164. doi:10.1094/pbiomes-12-18-0058-p

16. Dall'Asta P et al (2018) "Herbaspirillum Seropedicae Promotes Maize Growth but Fails to Control the Maize Leaf Anthracnose." Physiology and Molecular Biology of Plants, vol. 25, no. 1, pp. 167-176., doi:10.1007/s12298-018-0616-2

17. Doty SL, Oakley B, Xin G, Kang JW, Singleton G, Khan Z, Staley JT (2009) Diazotrophic endophytes of native black Cottonwood and willow. Symbiosis 47(1):23-33. doi:10.1007/bf03179967

18. Easlon HM, Bloom AJ (2014) "Easy Leaf Area: Automated Digital Image Analysis for Rapid and Accurate Measurement of Leaf Area." Applications in Plant Sciences, vol. 2, no. 7, p. 1400033. doi:10.3732/apps.1400033

19. Estrada GA (2012), pp. 115-129., Selection of Phosphate-Solubilizing Diazotrophic Herbaspirillum and Burkholderia Strains and Their Effect on Rice Crop Yield and Nutrient Uptake.. " Plant Soil 369:1-2. doi:10.1007/s11104-012-1550-7

20. FAOSTAT. (n.d.). Retrieved March 11 (2021) from http://www.fao.org/faostat/en/\#data/QC/visualize

21. Faville MJ, Briggs L, Cao M, Koulman A, Jahufer MZ, Koolaard J, Hume DE (2015) A QTL analysis of host Plant effects on fungal ENDOPHYTE biomass And alkaloid expression in perennial ryegrass. Mol Breeding 35(8). doi:10.1007/s11032-015-0350-1

22. Flint-Garcia SA, Thuillet A-C, Yu J, Pressoir G, Romero SM, Mitchell SE, Doebley J, Kresovich S, Goodman MM, Buckler ES (2005) Maize Association population: A High-resolution platform for quantitative trait locus dissection. Plant J 44(6):1054-1064. https://doi.org/10.1111/j.1365313x.2005.02591.x

23. Hungria $\mathrm{M}$ et al (2010) Inoculation with selected strains of Azospirillum brasilense and A. lipoferum improves yields of maize and wheat in Brazil. Plant and Soil. 331:413-4251-2): $\mathrm{p}$.

24. Khan Z, Rho H, Firrincieli A, Hung SH, Luna V, Masciarelli O, Doty SL (2016) Growth enhancement and drought tolerance of hybrid poplar upon inoculation with endophyte consortia. Current Plant Biology 6:38-47. doi:10.1016/j.cpb.2016.08.001

25. Kumara P, Mohana (2013) Endophytes and Plant Secondary Metabolite Synthesis: Molecular and Evolutionary Perspective.. " Advances in Endophytic Research 177-190. doi:10.1007/978-81-3221575-2_9 
26. Lin, F., Jiang, L., Liu, Y., Lv, Y., Dai, H., \& Zhao, H. (2014). Genome-wide identification of housekeeping genes in maize. Plant Molecular Biology, 86(4-5), 543-554. doi:10.1007/s11103-014-0246-1

27. Livak KJ, Schmittgen TD (2001) Analysis of relative gene expression data using real-time quantitative pcr and the $2-\delta \delta c t$ method. Methods 25(4):402-408. https://doi.org/10.1006/meth.2001.1262

28. Majeed A, Muhammad Z, Ahmad H (2018) Plant growth promoting bacteria: role in soil improvement, abiotic and biotic stress management of crops. Plant Cell Rep 37(12):1599-1609. https://doi.org/10.1007/s00299-018-2341-2

29. Matsumura EE, Secco VA, Moreira RS et al (2015) Composition and activity of endophytic bacterial communities in field-grown maize plants inoculated with Azospirillum brasilense. Ann Microbiol 65:2187-2200

30. Mcmullen MD (2009) Genetic Properties of the Maize Nested Association Mapping Population.. " Science 325(5941):737-740. doi:10.1126/science.1174320

31. Montanez A et al (2012) Characterization of cultivable putative endophytic plant growth promoting bacteria associated with maize cultivars (Zea mays L.) and their inoculation effects in vitro. Applied Soil Ecology. 58:: p. 21-28

32. Monteiro R, Adele et al (2012) "Herbaspirillum-Plant Interactions: Microscopical, Histological and Molecular Aspects." Plant and Soil, vol. 356, no. 1-2, pp. 175-196., doi:10.1007/s11104-012-1125-7

33. Mousa WK, Shearer CR, Limay-Rios V et al (2015) Bacterial endophytes from wild maize suppress Fusarium graminearum in modern maize and inhibit mycotoxin accumulation. Front Plant Sci 6:805

34. Naveed M (2014) Increased Drought Stress Resilience of Maize through Endophytic Colonization by Burkholderia Phytofirmans PsJN and Enterobacter Sp. FD17.. " Environ Exp Bot 97:30-39

35. Parnell JJ, Berka R, Young HA, Sturino JM, Kang Y, Barnhart DM, DiLeo MV (2016) From the lab to The Farm: An industrial perspective of plant Beneficial Microorganisms. Front Plant Sci 7. doi:10.3389/fpls.2016.01110

36. R Core Team (2021) R: A language and environment for statistical computing. R Foundation for Statistical Computing, Vienna, Austria. URL https://www.R-project.org/

37. Ray DK et al (2013) Yield Trends Are Insufficient to Double Global Crop Production by 2050. Plos One, 8(6)

38. Ray P (Dec. 2015) A Novel Delivery System for the Root Symbiotic Fungus, Sebacina Vermifera, and Consequent Biomass Enhancement of Low Lignin COMT Switchgrass Lines.. " Bioenergy Res 8(3):922-933. doi:10.1007/s12155-015-9636-8

39. Ray P (2018) Genome Sequence of the Plant Growth Promoting Fungus Serendipita Vermifera Subsp. Bescii: The First Native Strain from North America.. " Phytobiomes Journal 2(2):62-63. doi:10.1094/pbiomes-04-17-0017-a

40. Rho H, Hsieh M, Kandel SL, Cantillo J, Doty SL, Kim S (2017) Do endophytes promote growth of host plants under Stress? A meta-analysis on PLANT STRESS mitigation BY ENDOPHYTES. Microb Ecol 75(2):407-418. doi:10.1007/s00248-017-1054-3 
41. Riggs PJ (2001) Enhanced Maize Productivity by Inoculation with Diazotrophic Bacteria.. " Funct Plant Biol 28(9):829. doi:10.1071/pp01045

42. Rivas-Franco F (2020) Effects of a Maize Root Pest and Fungal Pathogen on Entomopathogenic Fungal Rhizosphere Colonization, Endophytism and Induction of Plant Hormones.. " Biol Control 150:104347. doi:10.1016/j.biocontrol.2020.104347

43. Rosenblueth M, Ormeño-Orrillo E, López-López A, Rogel MA, Reyes-Hernández BJ, Martínez-Romero JC, Reddy PM, Martínez-Romero E (2018) Nitrogen Fixation in Cereals. Frontiers in Microbiology, 9. https://doi.org/10.3389/fmicb.2018.01794

44. Shehata HR, Lyons EM, Jordan KS, Raizada MN (2016) Bacterial endophytes from wild and ancient maize are able to suppress the fungal pathogen Sclerotinia homoeocarpa. J Appl Microbiol 120:756-769

45. Shehata HR, Raizada MN (2017) A Burkholderia endophyte of the ancient maize landrace Chapalote utilizes c-di-GMP-dependent and independent signaling to suppress diverse plant fungal pathogen targets.FEMS Microbiol Lett364

46. Sobowale AA, Cardwell KF, Odebode AC et al (2007) Persistence of Trichoderma species within maize stem against Fusarium verticillioides. Arch Phytopathol Plant Prot 40:215-231

47. Timmusk S, Behers L, Muthoni J, Muraya A, Aronsson A (2017) Perspectives and challenges of microbial application for crop improvement. Front Plant Sci 8. doi:10.3389/fpls.2017.00049

48. Wallace JG (2018) and Georgiana May. "Endophytes: The Other Maize Genome."The Maize Genome, pp.213-246.

49. Walters WA et al (2018) "Large-Scale Replicated Field Study of Maize Rhizosphere Identifies Heritable Microbes." Proceedings of the National Academy of Sciences, vol. 115, no. 28, pp. 73687373., doi:10.1073/pnas.1800918115

50. Wang Y, Holland J, Balint-Kurti P (2021) Development and use of a seedling growth retardation assay to quantify and map loci underlying variation in the maize basal defense response. PhytoFrontiers ${ }^{\mathrm{TM}}$. doi:10.1094/phytofr-12-20-0038-r

51. Wintermans PC, Bakker PA, Pieterse CM (2016) Natural genetic variation in Arabidopsis for responsiveness to plant growth-promoting rhizobacteria. Plant Mol Biol 90(6):623-634. https://doi.org/10.1007/s11103-016-0442-2

52. Young L-S (2013) Endophytic Establishment of the Soil Isolate Burkholderia Sp. CC-Al74 Enhances Growth and P-Utilization Rate in Maize (Zea Mays L.).. " Appl Soil Ecol 66:40-47. doi:10.1016/j.apsoil.2013.02.001

53. Zhang X, Valdés-López O, Arellano C, Stacey G, Balint-Kurti P (2017) Genetic dissection of the MAIZE (zea Mays L.) mamp response. Theor Appl Genet 130(6):1155-1168. doi:10.1007/s00122-017-28766

\section{Figures}




\section{Figure 1}

ANOVA analysis. Standard ANOVA was used to break phenotypic variation into the effects of maize genotype, endophyte inoculation, their interaction, and residual error. Asterisks denote significant effects $(p<0.05)$. Plant genotype is almost always significant, while only Serendipita (Experiment 3 ) shows a consistent effect of the endophyte. Levene's test was used to check for equality of variances (one of the assumptions of ANOVA), and only Shoot Mass in Experiment 3 failed the test, so its results should be taken with caution.

\section{Figure 2}

Serendipita growth promotion

Effect of inoculation of S. bescii on different maize gentoypes for (A) shoot and (B) root biomass. Asterisks denote significant growth promotion. Several genotypes show growth promotion in only one of the two compartments; only P39 shows promotion in both.

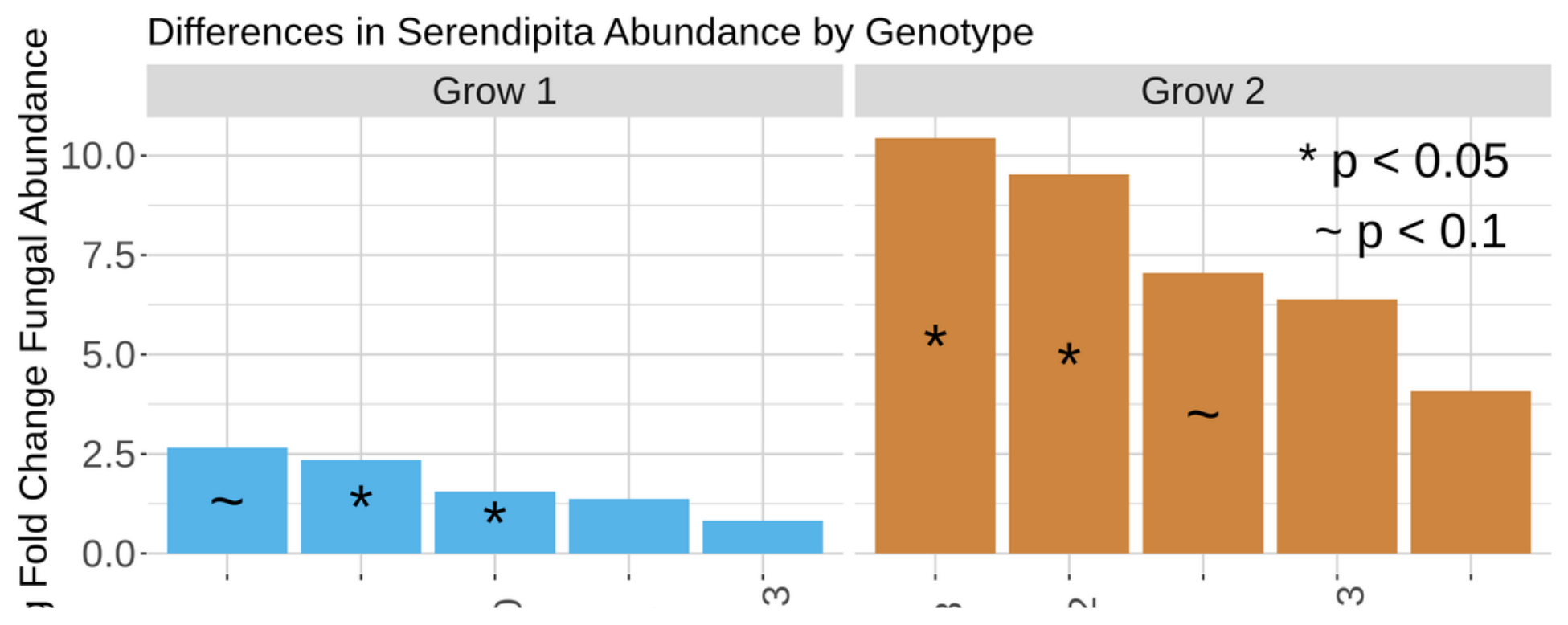

Figure 3

Differences in Serendipita Abundance by Genotype 
Log fold-change differences in S. bescii colonization between control and inoculated plants. The S. bescii ITS3 gene was quantified via qPCR and normalized to the maize CDK housekeeping gene. Since "grows" were planted on separate dates for space reasons, they are kept separate in this analysis. Within each grow, higher levels of colonization coincided with significant growth promotion (marked with $\sim$ or *). The least-colonized plants in each grow showed either no promotion or trends toward growth inhibition (Table 2).

\section{Image not available with this version}

\section{Figure 4}

This image is not available with this version.

\section{Supplementary Files}

This is a list of supplementary files associated with this preprint. Click to download.

- AdditionalFile1.csv

- CombinedRawqPCRclean.csv

- FinalBurkData.csv

- FinalHerbData.csv

- FinalSbecsiiData.csv 